\title{
POPULATION STUDY ARTICLE Higher chocolate intake is associated with longer telomere length among adolescents
}

\author{
Li Chen ${ }^{1}$, Haidong Zhu ${ }^{1}$, Bernard Gutin ${ }^{1}$, Howard D. Sesso ${ }^{2,3}$ and Yanbin Dong ${ }^{1}$
}

BACKGROUND: Chocolate intake has shown cardiometabolic health benefits. Whether chocolate has any effect on cellular aging remains unknown. We aimed to test the hypothesis that higher chocolate intake is associated with longer leukocyte telomere length (LTL) in adolescents.

METHODS: A total of 660 adolescents (aged 14-18 years) were included in the analysis. The chocolate intake was assessed by 7-day, 24-h dietary recalls and split into three groups, which were none, $<2$ servings/week, and 2 servings/week or more. LTL (T/S ratio) was determined by a modified quantitative polymerase chain reaction-based assay.

RESULTS: Among the 660 adolescents, $58 \%$ did not take any chocolate, $25 \%$ consumed $<2$ servings/week, and $17 \%$ consumed $\geq 2$ servings/week. Compared to non-consumers, adolescents who consumed chocolate of $\geq 2$ servings/week had 0.27 standard deviation (SD) longer LTL $(p=0.014)$. Higher chocolate consumption was associated with increased apolipoprotein A1 (ApoA1) $(p=0.038)$ and ApoA1/high-density lipoprotein (HDL) $(p=0.046)$. Moreover, higher ApoA1/HDL levels were correlated with longer LTL $(p=0.026)$.

CONCLUSION: Adolescents who consume 2 servings/week or more of chocolate candy have longer LTL compared with nonconsumers, and ApoA1/HDL pathway may be involved in this relationship.

Pediatric Research (2020) 87:602-607; https://doi.org/10.1038/s41390-019-0590-6

\section{INTRODUCTION}

Telomere is a special heterochromatic structure forming the end of linear chromosomes, which consists of GC-rich (guanine-cytosine-rich) repeated DNA sequences to maintain the chromosomal genomic stability. ${ }^{1}$ Telomeres are naturally shortened with every cell cycle. Therefore, telomere length implies the history of replication and is inversely related to chronological age, and is an emerging marker of cellular aging. ${ }^{1}$ We have previously shown that lifestyle factors, that is, physical inactivity and high dietary salt intake may influence leukocyte telomere length (LTL). ${ }^{2,3}$

Chocolate intake has been associated with potential cardiovascular benefits in recent studies, including lowering blood pressure ${ }^{4}$ and improving lipid profiles. ${ }^{5}$ These findings were recently reinforced by a meta-analysis of 14 prospective studies showing an inverse association between chocolate intake and cardiovascular disease (CVD) risk. ${ }^{6}$ However, whether chocolate intake has beneficial effect on cell aging process is not clear. Therefore, in this study, we aimed to test the hypothesis that higher chocolate candy consumption is associated with longer LTL in healthy adolescents. In addition, apolipoprotein, a lipoprotein metabolism regulator, is associated with chocolate intake in diabetic patients, ${ }^{7}$ and also related to LTL in adults aged 40 years or more. ${ }^{8}$ These findings imply a possible role of apolipoprotein in the association between chocolate intake and LTL. Thus, we also explored the involvement of apolipoprotein in the chocolate-telomere relationship.

\section{METHODS}

\section{Participants}

A cross-sectional cohort study was previously established in apparently healthy black and white adolescents aged 14 to 18 years old recruited from local public high schools in the Augusta, Georgia area, the southeastern region of the United States. ${ }^{9,10}$ Demographic data acquired from the education systems was utilized to select schools that enrolled both black and white students. Flyers were handed out to all students in the qualified schools with approvals from the county superintendents and school principals. The students who identified themselves as being white/ Caucasian or black/African American were qualified for the study. The students who responded were screened over telephone to verify their eligibility. The participants were excluded if they were taking current medications or diagnosed with chronic medical conditions that could affect growth, maturation, physical activity, nutritional status, or metabolism. All participants and the parents of participants who were minors provided written informed assent and consent prior to study participation. The Institutional Review Board at the Medical College of Georgia, Augusta University approved this study (Augusta, GA, protocol \#622505). A total of 660 participants (51\% girls, $48 \%$ blacks) with LTL and dietary assessment data available were included in the analysis.

\section{Dietary assessment}

As previously described, ${ }^{9,10}$ 7-day non-consecutive, 24-h dietary recalls were collected using the Nutrition Data System for Research (NDS-R 2006, Nutrition Coordinating Center, University

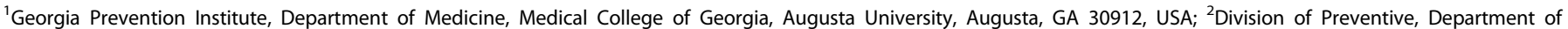
Medicine, Brigham and Women's Hospital, Boston, MA 02215, USA and ${ }^{3}$ Department of Epidemiology, Harvard T.H. Chan School of Public Health, Boston, MA 02115, USA Correspondence: Yanbin Dong (YDONG@augusta.edu)
} 
of Minnesota, Minneapolis, MN) by trained dietitians. Seven separate 24-h dietary recalls were collected during a period of 12 weeks for each participant. Among the seven recalls, five took place at weekdays, and two took place at weekends, which captured the variability. The first two were carried out in person and the remaining five were collected over the telephone. The participants were not told of the telephone recall schedule to reduce possible changes in eating patterns. Adolescents who had only three recalls or less (5.5\%) were excluded. Fifty-two percent of adolescents had recalls for all 7 days.

An average chocolate intake from four to seven recalls was calculated and split into three groups: none, $<2$ servings/week, and $\geq 2$ servings/week. One serving of chocolate candy equals to $40 \mathrm{~g}$. Food models, portion booklets, and serving containers were used to assist in estimating the serving size. Dietary quality was measured by the 2010 update of the Alternate Healthy Eating Index (AHEl-2010), based on foods and nutrients predictive of chronic disease risk ${ }^{11}$ and updated to incorporate recent scientific evidence. ${ }^{12}$ The components comprising the score include vegetables, fruit, whole grains, sugar-sweetened beverages and fruit juice, nut and legumes, red/processed meat, trans fat, longchain $(n-3)$ fats, polyunsaturated fatty acids, sodium, and alcohol. The AHEI-2010 score ranges from 0 to $110 .{ }^{10}$

Physical activity

Physical activity level was assessed using MTI Actigraph monitors (model 7164; MTIHealth Services, Fort Walton Beach, FL), uniaxial accelerometers that measure vertical acceleration and deceleration. The participants were directed to wear the monitor for 7 continuous days. Records from days 1 to 7 were excluded because a full day of information was not available for those days. Average daily time ( $\mathrm{min}$ ) spent in moderate (3-6 metabolic equivalents) and vigorous (6 metabolic equivalents) physical activity was calculated from the movement counts by the software accompanying the device.

\section{Anthropometry measurements}

Height was measured twice to the nearest $0.1 \mathrm{~cm}$ by a wallmounted stadiometer (Tanita Corporation of American, Arlington Heights, IL); weight was measured twice to the nearest $0.1 \mathrm{~kg}$ by a calibrated electronic scale with the participants not wearing shoes and in light clothing (model CN2OL; Cardinal Detecto, Webb City, $\mathrm{MO}$ ). Body mass index (BMI) was computed as weight (in $\mathrm{kg}$ ) per square of height (in $\mathrm{m}^{2}$ ). BMI percentile was calculated based on the 2000 CDC Growth Charts for the United States. Sexual development of the participants was measured by a five-stage scale, ranging from 1 (prepubertal) to 5 (fully mature) as previously described. ${ }^{10,13}$ Using a sex-specific questionnaire, the participants reported their sexual maturation stage by comparing their own physical development to the five stages in standard sets of diagrams. A parent or research coordinator then went over the results with the youths to ensure that they understood the questionnaire. When a participant reported discordant stages of pubic hair and breast or genital development, the higher one was used. Self-assessed sexual maturation has shown reasonable validity against physician assessments and hormone levels, and has been employed in epidemiologic researches for evaluating sexual maturation in children. ${ }^{14}$

\section{Family socioeconomic status estimate}

Hollingshead Four-Factor Social Status Index was calculated by a function, including the parental education level, employment status, and occupation, with a greater value indicating a higher socioeconomic status (SES). ${ }^{15}$ The child participants' parents' education is rated on a 7-point scale that lists the highest grade completed, and the occupational is rated on a 9-point scale. SES was then computed by multiplying the occupation scale value by a weight of 5 and the education scale value by 3 and summing the products. Hollingshead Index scores ranged from 8 to 66 .

Measurement of lipid and apolipoprotein

Plasma high-density lipoprotein (HDL)-cholesterol concentrations were quantified at the Emory Lipid Research Laboratory using homogeneous enzymatic assays (Equal Diagnostics, Exton, PA). Plasma concentrations of apolipoprotein $A 1$ (ApoA1) and ApoB were quantified using immunoturbidimetric methods (DiaSorin, Stillwater, MN). ApoC-III was quantified using an Immunoturbidometric Assay Kit (Wako Chemicals, Richmond, VA). ApoE was measured on the Beckman CX7 Chemistry analyzer by immunoturbidometric method using the kit from Wako Chemicals (Richmond, VA).

\section{Measurement of LTL}

Mean LTL was measured from leukocyte DNA by a modified quantitative polymerase chain reaction (PCR)-based assay as previously described. ${ }^{3,13}$ Briefly, the telomere repeat copy number (T) and single-copy gene copy number (36B4 gene, S) were measured by a 7500 Fast Realtime PCR System (Applied Biosystems, Foster City, CA). Samples were processed in triplicate. Threshold values $(\mathrm{Ct})$ were calculated by averaging the triplicates. Each 96-well plate contained a 5-point standard curve using the same control genomic DNA from 3 to $48 \mathrm{ng}$. Telomere PCRs and 36B4 PCRs were performed on separate plates, with the same sample well position. T/S ratio was calculated as the amount of telomeric DNA $(\mathrm{T})$ divided by the amount of single-copy control gene DNA (S). The intra-plate and inter-plate coefficients of variation for the $\mathrm{T} / \mathrm{S}$ ratio were $5.6 \%$ and $6.8 \%$, respectively.

\section{Statistical analyses}

The baseline characteristics of the participants are summarized by mean \pm standard deviation (SD) for continuous variables and $N(\%)$ for categorical variables. Normality of each continuous variable was tested based on a combination of skewness and kurtosis. Univariate analyses testing the difference of continuous measurements among different chocolate intake groups was conducted using analysis of variance for normally distributed variables or by Kruskal-Wallis test, otherwise. $x^{2}$ tests were conducted for categorical variables. Linear regression models were used to calculate the associations between chocolate intake, LTL, and apolipoprotein. LTL was standardized to zero-mean and unitvariance before regression. Three models with different covariates were performed. Model 1 was adjusted for age, sex, race, and BMI percentile. Model 2 additionally adjusted for energy intake and AHEI-2010. Finally, Model 3 additionally adjusted for nonchocolate candy intake, physical activity, family SES, and sexual development. A $p$ value $<0.05$ was considered statistically significant. All statistical analyses were performed using Stata version 12.0 (College Station, TX).

\section{RESULTS}

Demographics and univariate analysis

Among 660 adolescents aged $16.1 \pm 1.2$ years, 383 (58\%) did not consume any chocolate during the dietary recall period, $165(25 \%)$ consumed $<2$ servings/week of chocolate, and $122(17 \%)$ consumed $\geq 2$ servings/week of chocolate. BMI percentile was inversely associated with the chocolate intake $(p<0.001)$. Adolescents who consumed more chocolate also consumed greater calories $(p=0.005)$ and non-chocolate candy $(p<0.001)$. The proportion of non-consumers among boys $(66 \%)$ was significantly higher than girls $(51 \%, p<0.001)$, and blacks $(64 \%)$ were higher than whites $(53 \%, p=0.007)$. The differences in age, sexual development, family SES, physical activity, AHEI, and apolipoprotein among chocolate intake groups were not significant (Table 1). 
Higher chocolate intake is associated with longer telomere length among...

$L$ Chen et al.

Table 1. General characteristics of adolescents stratified by chocolate intake group

\begin{tabular}{|c|c|c|c|c|c|}
\hline Demographics & Total & \multicolumn{3}{|c|}{ Chocolate intake } & $p$ Value \\
\hline$N(\%)$ & $660(100)$ & $383(58)$ & $165(25)$ & $112(17)$ & - \\
\hline Age (years) & $16.1 \pm 1.2$ & $16.2 \pm 1.2$ & $16.0 \pm 1.2$ & $16.1 \pm 1.3$ & 0.104 \\
\hline Sex & & & & & $<0.001$ \\
\hline Male (\%) & $322(49)$ & $212(55)$ & $60(36)$ & $50(45)$ & \\
\hline White (\%) & $346(52)$ & $183(48)$ & $103(62)$ & $60(54)$ & \\
\hline Black (\%) & $314(48)$ & $200(52)$ & $62(38)$ & $52(46)$ & \\
\hline BMI $\left(\mathrm{kg} / \mathrm{m}^{2}\right)$ & $23.0 \pm 4.9$ & $23.5 \pm 5.2$ & $22.8 \pm 5.0$ & $21.3 \pm 3.6$ & $<0.001$ \\
\hline BMI percentile & $0.5 \pm 0.3$ & $0.5 \pm 0.3$ & $0.5 \pm 0.3$ & $0.4 \pm 0.3$ & $<0.001$ \\
\hline Sexual development & $4.3 \pm 0.7$ & $4.4 \pm 0.8$ & $4.4 \pm 0.7$ & $4.3 \pm 0.7$ & 0.444 \\
\hline Non-chocolate candy intake (servings/week) & $0.8 \pm 2.1$ & $0.7 \pm 2.2$ & $0.6 \pm 1.2$ & $1.7 \pm 2.3$ & $<0.001$ \\
\hline Energy Intake (kcal/day) & $1945 \pm 595$ & $1910 \pm 616$ & $1937 \pm 567$ & $2081 \pm 546$ & 0.005 \\
\hline AHEI-2010 & $35.1 \pm 8.8$ & $35.2 \pm 8.7$ & $34.7 \pm 8.1$ & $35.2 \pm 10.1$ & 0.970 \\
\hline \multicolumn{6}{|l|}{ Apolipoprotein } \\
\hline ApoA1 (mg/dL) & $120 \pm 26$ & $119 \pm 26$ & $121 \pm 24$ & $124 \pm 25$ & 0.082 \\
\hline ApoA1/HDL & $2.6 \pm 0.5$ & $2.6 \pm 0.6$ & $2.6 \pm 0.5$ & $2.6 \pm 0.5$ & 0.335 \\
\hline ApoB (mg/dL) & $69.6 \pm 17.9$ & $70.0 \pm 18.2$ & $69.9 \pm 17.5$ & $67.8 \pm 17.7$ & 0.689 \\
\hline ApoC-III (ng/mL) & $12.4 \pm 4.0$ & $12.2 \pm 3.9$ & $12.9 \pm 4.0$ & $12.1 \pm 3.9$ & 0.103 \\
\hline ApoE (ng/mL) & $4.1 \pm 1.4$ & $4.1 \pm 1.3$ & $4.2 \pm 1.6$ & $4.1 \pm 1.4$ & 0.932 \\
\hline
\end{tabular}

Note: Statistics display as mean \pm SD for continuous variables, and $N(\%)$ for categorical variables

$B M I$ body mass index, SES socioeconomic status, MET metabolic equivalent, AHEI Alternate Healthy Eating Index

Telomere length by chocolate intake groups

Figure 1 compares LTL according to chocolate intake groups. Higher chocolate consumption was significantly associated with longer LTL $(p=0.018)$. For chocolate consumption of none, $<2$ servings/week and $\geq 2$ servings/week, the average LTL were $1.28 \pm 0.22,1.28 \pm 0.25$ and $1.33 \pm 0.22$, respectively.

Association between chocolate intake and telomere length Compared to the non-consumers of chocolate, adolescents who consumed $\geq 2$ servings/week of chocolate had 0.27 SD longer LTL $(p=0.014)$ in Model 1 adjusting for age, sex, race, and BMI percentile. The significant association persisted, with no appreciated reduction in the effect size, after additional adjustment of energy intake and AHEI-2010 in Model $2(\beta=0.28, p=0.011)$, and non-chocolate candy intake, physical activity, family SES, and sexual development in Model 3 ( $\beta=0.27, p=0.039$ ) (Table 2). We found no significant interaction between chocolate intake and race or sex on LTL.

Associations between chocolate candy intake and apolipoprotein In Table 3, compared to the non-consumers of chocolate, adolescents who consumed $\geq 2$ servings $(80 \mathrm{~g}) /$ week of chocolate had a higher level of ApoA1 $(\beta=0.06, p=0.038)$ in Model 3. There was no significant association between the chocolate intake and other apolipoproteins, which included ApoB, ApoC-III, and ApoE ( $\left.p^{\prime} s>0.05\right)$. In addition, Table 4 shows that chocolate intake was also associated with ApoA1/HDL $(\beta=0.04, p=0.046)$ in Model 1 . The associations in Models 2 and 3 were not significant, but at borderline in Model $2(p=0.067)$.

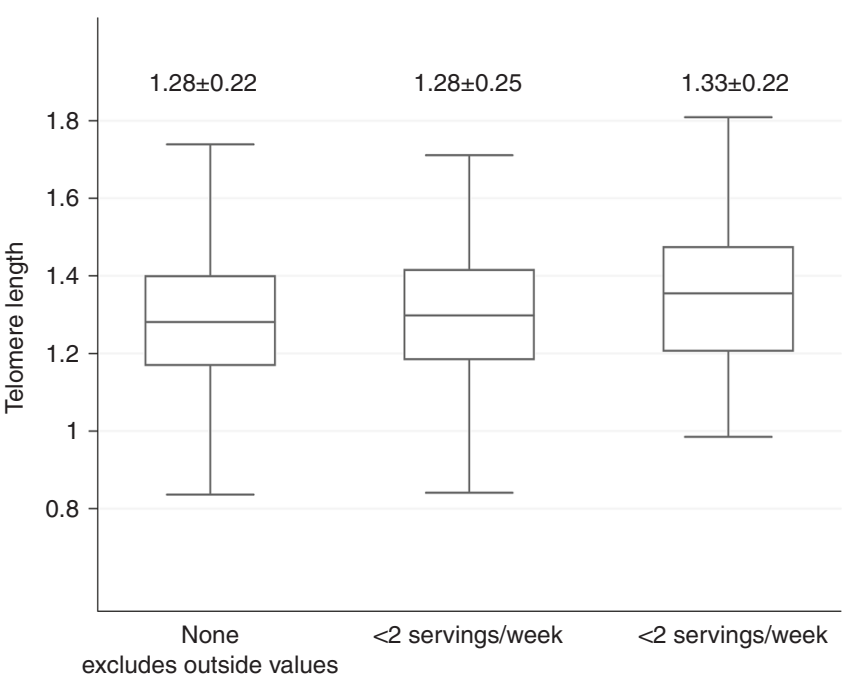

Fig. 1 Box plots of telomere length over chocolate intake group. Median, 25th, and 75th percentiles of LTL are presented in the box plots. Mean \pm SD is tagged on top of each box

Associations between ApoA1, ApoA1/HDL, and telomere length A higher level of ApoA1, which was associated with more chocolate consumption, was also associated with longer $\operatorname{LTL}(\beta=0.44, p=$ 0.049 ) in Model 3. In addition, ApoA1/HDL was positively correlated to LTL in all three models with $p^{\prime} s$ of $0.026,0.018$, and 0.003 , respectively (Table 4 ). 
Table 2. Adjusted associations between chocolate intake groups and telomere length

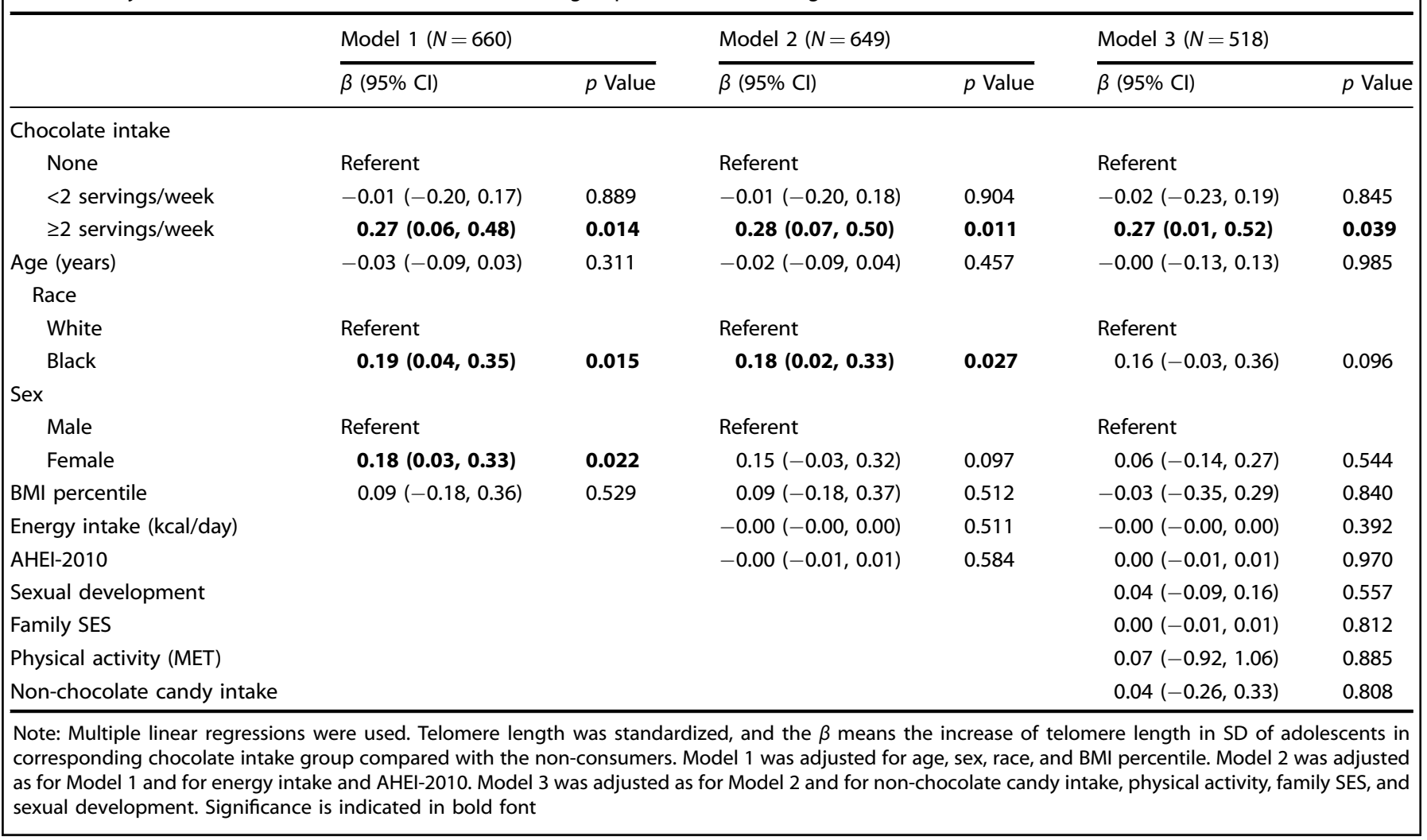

Table 3. Adjusted associations between chocolate intake groups and apolipoprotein

\begin{tabular}{|c|c|c|c|c|c|c|}
\hline & \multicolumn{2}{|l|}{ Model 1} & \multicolumn{2}{|l|}{ Model 2} & \multicolumn{2}{|l|}{ Model 3} \\
\hline \multicolumn{7}{|c|}{ Independent var:: Chocolate intake } \\
\hline None & Referent & & Referent & & Referent & \\
\hline$<2$ servings/week & $0.01(-0.03,0.05)$ & 0.562 & $0.01(-0.03,0.05)$ & 0.543 & $0.01(-0.03,0.06)$ & 0.596 \\
\hline \multicolumn{7}{|c|}{ Independent var.: Chocolate intake } \\
\hline None & Referent & & Referent & & Referent & \\
\hline$<2$ servings/week & $0.03(-0.02,0.07)$ & 0.310 & $0.03(-0.02,0.08)$ & 0.204 & $0.02(-0.04,0.07)$ & 0.521 \\
\hline$\geq 2$ servings/week & $-0.01(-0.07,0.05)$ & 0.747 & $0.01(-0.05,0.07)$ & 0.863 & $-0.00(-0.07,0.07)$ & 0.971 \\
\hline Dependent var:: ApoC-III & $(N=642)$ & & $(N=629)$ & & $(N=509)$ & \\
\hline$\geq 2$ servings/week & $0.01(-0.06,0.08)$ & 0.756 & $0.02(-0.06,0.10)$ & 0.590 & $0.01(-0.08,0.10)$ & 0.822 \\
\hline Dependent var.: ApoE & $(N=651)$ & & $(N=638)$ & & $(N=518)$ & \\
\hline \multicolumn{7}{|c|}{ Independent var.: Chocolate intake } \\
\hline None & Referent & & Referent & & Referent & \\
\hline$<2$ serings/week & $0.00(-0.05,0.06)$ & 0.926 & $0.01(-0.05,0.06)$ & 0.783 & $0.02(-0.04,0.08)$ & 0.536 \\
\hline$\geq 2$ servings/week & $0.01(-0.05,0.08)$ & 0.736 & $0.02(-0.05,0.09)$ & 0.506 & $0.05(-0.03,0.13)$ & 0.189 \\
\hline
\end{tabular}


Table 4. Adjusted associations between chocolate intake groups, telomere length, ApoA1, and ApoA1/HDL

\begin{tabular}{|c|c|c|c|c|c|c|}
\hline & \multicolumn{2}{|l|}{ Model 1} & \multicolumn{2}{|l|}{ Model 2} & \multicolumn{2}{|l|}{ Model 3} \\
\hline Independent var:: ApoA1 & $0.23(-0.15,0.62)$ & 0.233 & $0.25(-0.14,0.64)$ & 0.208 & $0.44(0.00,0.89)$ & 0.049 \\
\hline Dependent var.: LTL & $(N=600)$ & & $(N=589)$ & & $(N=474)$ & \\
\hline Independent var.: ApoA1/HDL & $0.50(0.06,0.93)$ & 0.026 & $0.53(0.09,0.97)$ & 0.018 & $0.76(0.26,1.25)$ & 0.003 \\
\hline \multicolumn{7}{|c|}{ Independent var:: Chocolate intake } \\
\hline None & Referent & & Referent & & Referent & \\
\hline$<2$ servings/week & $-0.00(-0.04,0.03)$ & 0.911 & $-0.00(-0.04,0.03)$ & 0.885 & $-0.01(-0.05,0.03)$ & 0.679 \\
\hline$\geq 2$ servings/week & $0.04(0.00,0.08)$ & 0.046 & $0.04(-0.00,0.08)$ & 0.067 & $0.04(-0.01,0.09)$ & 0.120 \\
\hline
\end{tabular}

\section{DISCUSSION}

Our results show that the adolescents who consume 2 servings/ week or more of chocolate candy have longer LTL than those not reporting chocolate intake. In addition, chocolate candy intake was positively associated with ApoA 1 and ApoA1/HDL. ApoA 1 and ApoA1/HDL were further associated with LTL.

LTL is a key biomarker of cellular aging and plays a role in cardiovascular health. Shorter LTL has been associated with a greater risk of coronary artery disease ${ }^{16}$ and coronary artery calcification. ${ }^{17}$ LTL is also inversely associated with blood pressure, ${ }^{18}$ pulse wave velocity, ${ }^{19}$ and intima-media thickness. ${ }^{20}$ Although no studies have examined chocolate intake and LTL, a healthy dietary pattern is considered to have a positive impact on LTL. Better adherence to the Mediterranean diet has been associated with longer LTL in healthy women. ${ }^{21}$ Another study reported that better adherence to the Mediterranean diet was associated with longer LTL, but not among African Americans and Hispanics. ${ }^{22}$ An anti-inflammatory diet appears to slow down telomere shortening among populations at high CVD risk in a study with 5 years of follow-up. ${ }^{23}$ Longer LTL has also been related to higher dietary antioxidant capacity and lower white bread consumption in Spanish children and adolescents. ${ }^{24}$ We have previously shown that girls who engage in more vigorous physical activity had longer LTL. ${ }^{3}$ In addition, overweight/obese adolescents consumed more dietary sodium had shorter LTL. ${ }^{2}$

In a longitudinal study of the general population, one SD longer LTL at baseline was independently associated with an $11 \%$ less mortality risk. ${ }^{25}$ Another cohort study of patients with stable coronary heart disease found that each SD increase in LTL was related to a $24 \%$ reduction in mortality. ${ }^{26}$ In this study, we found that adolescents who consumed $\geq 2$ servings/week of chocolate candy had $0.27 \mathrm{SD}$ longer LTL compared to the non-consumers of chocolate, which may have clinical significance.

Flavanol and theobromine are the most commonly studied nutrients underlying any potential health effects of chocolate based on its cocoa content. Dark chocolate has higher cocoa content and tends to contain more flavanols, which have antioxidant properties that may prevent DNA damage and improve the nucleus integrity of cells. ${ }^{27}$ Flavanols upregulate endothelial nitric oxide (NO)-synthase activity through the insulin-mediated signaling pathway $^{28}$ and induce NO-dependent vasodilation, ${ }^{29}$ whereas the inhibition of NO synthase accelerates the shortening of LTL. ${ }^{30}$ Flavanols also appear to inhibit angiotensin-converting enzyme (ACE) activity, ${ }^{28}$ whereby the ACE deletion genotype has been shown to have a negative effect on $\mathrm{LTL}^{31}$ In a randomized clinical trial testing daily intake of $2 \mathrm{~g}$ dark chocolate with $70 \%$ cocoa for 6 months, there were significant improvements in the lipid profile. ${ }^{27}$ High flavanol and high theobromine chocolate also decreases arterial stiffness in pregnant women compared to low flavonol and low theobromine chocolate. ${ }^{32}$

Apolipoprotein regulates lipoprotein metabolism through its function of transport and redistribution of lipid, cofactors for enzymes of lipid metabolism, and maintenance of the structure of the lipoprotein particles. ${ }^{33}$ Therefore, apolipoprotein is associated with lipid profiles ${ }^{34}$ and cardiovascular health. ${ }^{35}$ ApoA1 is a main protein moiety in HDL particles. Low serum ApoA1 level is a risk factor of CVD. ${ }^{36}$ ApoA1/HDL fraction in serum is indicative in the removal of excessive cholesterol and phospholipids from ABCA1-expressing cells, thereby preventing the formation of foam cells, which are key contributors to the atheroma formation. ${ }^{37}$ We found that higher chocolate candy consumption was associated with a higher level of ApoA1 and higher ApoA1/HDL ratio in adolescents. A previous trial also found that serum levels of ApoA1 were increased after 8-week dark chocolate consumption compared with baseline among 32 diabetic patients. ${ }^{7}$ A positive association among ApoA1 and LTL was reported among adults aged 40 years or more. ${ }^{8}$ We also found positive associations among ApoA1, ApoA1/HDL and LTL in adolescents, which supported the hypothesis that ApoA1/HDL pathway could mediate the association between chocolate consumption and LTL.

Greenberg et al. ${ }^{38}$ have argued that the significant associations between greater chocolate consumption and favorable health outcomes may be partially explained by reverse causation, with reductions in chocolate consumption that follow after a disease diagnosis. A meta-analysis found that chocolate consumption, nonchocolate candy consumption, and total confectionery consumption were all significantly and inversely associated with obesity. ${ }^{39}$ The study suggested that overweight or obese youth would reduce their confectionery intake more than non-overweight youth if the youth or parents believed that it was contributing toward their obesity. ${ }^{39}$ Our findings of a beneficial effect of chocolate intake on LTL may be less influenced by reverse causality since LTL is unlikely to affect a decision to consume chocolate. However, similarly, the overweight or obese adolescents may be restricted on chocolate intake, as we observed an inverse association between BMI percentile and chocolate consumption. On the other hand, the inverse association between the chocolate intake and LTL was still significant after adjustment of BMI percentile. 
To the best of our knowledge, this study is the first to report a positive association between chocolate intake and LTL. Limitations of our study should be recognized. First, causal relationship cannot be established from the cross-sectional study. Second, our dietary intake assessment did not distinguish among the different types of chocolate and was unable to quantify the effective nutrients, such as flavanols. In addition, other food items containing chocolate, such as chocolate cake and chocolate milk, were not included in this study due to the limitation of the Nutrition Data System for Research.

In conclusion, adolescents consuming 2 or more servings/week of chocolate have longer LTL compared with those consuming no chocolate, and ApoA1/HDL pathway could be involved in this association. Randomized clinical trials are warranted to establish the beneficial effect of chocolate on cellular aging processes in the future.

\section{ACKNOWLEDGEMENTS}

This work was funded by the National Institute of Health, grant number HL064157.

\section{AUTHOR CONTRIBUTIONS}

L.C. and Y.D. proposed the study idea and drafted the initial manuscript. H.Z., B.G. H.D.S., and Y.D. conceptualized and designed the study, collected the data, and critically reviewed and revised the manuscript. All authors approved the final manuscript as submitted and agree to be accountable for all aspects of the work.

\section{ADDITIONAL INFORMATION}

Competing interests: H.D.S. declares that he has received investigator-initiated grant support from Mars Symbioscience and Pfizer Inc. (including donations of study pills) for the COcoa Supplement and Multivitamin Outcomes Study (COSMOS). All other authors have indicated they have no financial relationships relevant to this article to disclose.

Publisher's note Springer Nature remains neutral with regard to jurisdictional claims in published maps and institutional affiliations.

\section{REFERENCES}

1. Benetos, A. et al. Telomere length as an indicator of biological aging: the gender effect and relation with pulse pressure and pulse wave velocity. Hypertension 37, 381-385 (2001)

2. Zhu, H. et al. High sodium intake is associated with short leukocyte telomere length in overweight and obese adolescents. Int J. Obes. (Lond.) 39, 1249-1253 (2015).

3. Zhu, $\mathrm{H}$. et al. Leukocyte telomere length in healthy Caucasian and AfricanAmerican adolescents: relationships with race, sex, adiposity, adipokines, and physical activity. J. Pediatr. 158, 215-220 (2011).

4. Grassi, D. et al. Flavanol-rich chocolate acutely improves arterial function and working memory performance counteracting the effects of sleep deprivation in healthy individuals. J. Hypertension 34, 1298-1308 (2016).

5. Fernandez-Murga, L., Tarin, J. J., Garcia-Perez, M. A. \& Cano, A. The impact of chocolate on cardiovascular health. Maturitas 69, 312-321 (2011).

6. Ren, Y. et al. Chocolate consumption and risk of cardiovascular diseases: a metaanalysis of prospective studies. Heart 105, 49-55 (2019).

7. Rostami, A. et al. High-cocoa polyphenol-rich chocolate improves blood pressure in patients with diabetes and hypertension. ARYA Atheroscler. 11, 21-29 (2015).

8. Panayiotou, A. G. et al. Leukocyte telomere length is associated with measures of subclinical atherosclerosis. Atherosclerosis 211, 176-181 (2010).

9. Chen, L. et al. Epigenome-Wide Association Study of dietary fiber intake in African American adolescents. Mol. Nutr. Food Res. 62, e1800155 (2018).

10. Stallmann-Jorgensen, I. S. et al. General and visceral adiposity in black and white adolescents and their relation with reported physical activity and diet. Int. J. Obes. 31, 622-629 (2007)

11. McCullough, M. L. et al. Diet quality and major chronic disease risk in men and women: moving toward improved dietary guidance. Am. J. Clin. Nutr. 76, 1261-1271 (2002).

12. Chiuve, S. E. et al. Alternative dietary indices both strongly predict risk of chronic disease. J. Nutr. 142, 1009-1018 (2012).
13. Dong, Y. et al. Associations between global DNA methylation and telomere length in healthy adolescents. Sci. Rep. 7, 4210 (2017).

14. Chavarro, J. E. et al. Validity of self-assessed sexual maturation against physician assessments and hormone levels. J. Pediatr. 186, 172-178.e173 (2017).

15. Hollingshead, A. B. 1975 Four factor index of social status. Unpublished manuscript, Yale University, New Haven, CT (1975)

16. Hamad, R., Walter, S. \& Rehkopf, D. H. Telomere length and health outcomes: A two-sample genetic instrumental variables analysis. Exp. Gerontol. 82, 88-94 (2016).

17. Mwasongwe, S. et al. Leukocyte telomere length and cardiovascular disease in African Americans: The Jackson Heart Study. Atherosclerosis 266, 41-47 (2017).

18. Raymond, A. R. et al. Telomere length, endothelial activation and carotid atherosclerosis in black and white African patients with rheumatoid arthritis. Clin. Exp. Rheumatol. 34, 864-871 (2016).

19. Raymond, A. R., Norton, G. R., Woodiwiss, A. J. \& Brooksbank, R. L. Impact of gender and menopausal status on relationships between biological aging, as indexed by telomere length, and aortic stiffness. Am. J. Hypertens. 28, 623-630 (2015).

20. Spigoni, V. et al. Telomere length is independently associated with subclinical atherosclerosis in subjects with type 2 diabetes: a cross-sectional study. Acta Diabetol. 53, 661-667 (2016).

21. Crous-Bou, M. et al. Mediterranean diet and telomere length in Nurses' Health Study: population based cohort study. BMJ 349, g6674 (2014).

22. Gu, Y. et al. Mediterranean diet and leukocyte telomere length in a multi-ethnic elderly population. Age (Dordr.) 37, 24 (2015).

23. Garcia-Calzon, S. et al. Dietary inflammatory index and telomere length in subjects with a high cardiovascular disease risk from the PREDIMED-NAVARRA study: crosssectional and longitudinal analyses over 5 y. Am. J. Clin. Nutr. 102, 897-904 (2015).

24. Garcia-Calzon, S. et al. Dietary total antioxidant capacity is associated with leukocyte telomere length in a children and adolescent population. Clin. Nutr. 34, 694-699 (2015).

25. Marioni, R. E. et al. The epigenetic clock and telomere length are independently associated with chronological age and mortality. Int. J. Epidemiol. 45, 424-432 (2016).

26. Goglin, S. E. et al. Change in leukocyte telomere length predicts mortality in patients with stable coronary heart disease from the heart and soul study. PLOS ONE 11, e0160748 (2016).

27. Leyva-Soto, A., Chavez-Santoscoy, R. A., Lara-Jacobo, L. R., Chavez-Santoscoy, A. V., Gonzalez-Cobian, L. N. Daily consumption of chocolate rich in flavonoids decreases cellular genotoxicity and improves biochemical parameters of lipid and glucose metabolism. Molecules 23, 2220 (2018).

28. Ried, K., Fakler, P. \& Stocks, N. P. Effect of cocoa on blood pressure. Cochrane Database Syst. Rev. 4, CD008893 (2017).

29. Mogollon, J. A. et al. Blood pressure and endothelial function in healthy, pregnant women after acute and daily consumption of flavanol-rich chocolate: a pilot, randomized controlled trial. Nutr. J. 12, 41 (2013).

30. Scalera, F. et al. Endogenous nitric oxide synthesis inhibitor asymmetric dimethyl L-arginine accelerates endothelial cell senescence. Arterioscler Thromb. Vasc. Biol. 24, 1816-1822 (2004).

31. Fyhrquist, F. et al. Telomere length is associated with ACE I/D polymorphism in hypertensive patients with left ventricular hypertrophy. J. Renin Angiotensin Aldosterone Syst. 14, 227-234 (2013).

32. Babar, A. et al. Changes in endothelial function, arterial stiffness and blood pressure in pregnant women after consumption of high-flavanol and hightheobromine chocolate: a double blind randomized clinical trial. Hypertens. Pregnancy 37, 68-80 (2018).

33. Mahley, R. W., Innerarity, T. L., Rall, S. C. Jr. \& Weisgraber, K. H. Plasma lipoproteins: apolipoprotein structure and function. J. Lipid Res. 25, 1277-1294 (1984).

34. Yamauchi, K., Ebihara, Y. \& Kawakami, Y. Redox status of serum apolipoprotein E and its impact on HDL cholesterol levels. Clin. Biochem. 50, 777-783 (2017).

35. Zhu, Y. M. et al. Association of Apolipoproteins B and A-1 With Markers of Vascular Health or Cardiovascular Events. Can. J. Cardiol. 33, 1305-1311 (2017).

36. Frondelius, K. et al. Lifestyle and dietary determinants of serum apolipoprotein A1 and apolipoprotein B concentrations: cross-sectional analyses within a swedish cohort of 24,984 individuals. Nutrients 9, 211 (2017).

37. Chistiakov, D. A., Orekhov, A. N. \& Bobryshev, Y. V. ApoA1 and ApoA1-specific selfantibodies in cardiovascular disease. Lab Invest. 96, 708-718 (2016)

38. Greenberg, J. A. et al. Chocolate intake and heart disease and stroke in the Women's Health Initiative: a prospective analysis. Am. J. Clin. Nutr. 108, 41-48 (2018).

39. Gasser, C. E., Mensah, F. K., Russell, M., Dunn, S. E. \& Wake, M. Confectionery consumption and overweight, obesity, and related outcomes in children and adolescents: a systematic review and meta-analysis. Am. J. Clin. Nutr. 103, 1344-1356 (2016). 